\title{
The Relationship Among College Students' Internet Self-Disclosure, Self - Esteem and Mental Health
}

\author{
Zhang Yu ${ }^{1,2}$, Li Pengsong ${ }^{1}$, Ye Mao ${ }^{3}$, Cao Houwen ${ }^{1}$, Song Ge $^{1}$, Zhu Zhennan ${ }^{1}$, Zhao Zhinan ${ }^{1}$, \\ $\mathrm{Li} \mathrm{Na}^{4}$, \\ ${ }^{1}$ Department of Physical Education, Dalian University of Technology, Dalian, China \\ ${ }^{2}$ State Key Laboratory of Fine Chemicals, Dalian R\&D Center for Stem Cell and Tissue Engineering, Dalian University of Technology, Dalian, \\ China \\ ${ }^{3}$ Xianning Central Hospital, Dalian Medical University, Dalian, China \\ ${ }^{4}$ Dalian Medical University, Dalian, China
}

\section{Email address:}

zhangyu0705@dlut.edu.cn (Zhang Yu),Li70@dlut.edu.cn (Li Pengsong),282124010@qq.com (Cao Houwen), songge@dlut.edu.cn (Song Ge), zhuzn@dlut.edu.cn (Zhu Zhennan), zhaozn@dlut.edu.cn (Zhao Zhinan), 55286747@qq.com (Li Na)

${ }^{*}$ Corresponding author

\section{To cite this article:}

Zhang Yu, Li Pengsong, Ye Mao, Cao Houwen, Song Ge, Zhu Zhennan, Zhao Zhinan, Li Na. The Relationship Among College Students' Internet Self-Disclosure, Self - Esteem and Mental Health. Humanities and Social Sciences. Vol. 5, No. 2, 2017, pp. 84-90. doi: $10.11648 /$ j.hss.20170502.16

Received: January 13, 2017; Accepted: April 18, 2017; Published: April 21, 2017

\begin{abstract}
This study was to explore the relationship between psychological development of college students and the degree of self-disclosure on social networks from two aspects of self-esteem and mental health. Through questionnaire analysis, analyzed 172 college students' social web page (take Renren as example) self-disclosure (including the number of friends, published state, log, upload photos and text reply information etc.) correlated with self-esteem and mental health. Results: there was a significant difference between male and female college students in the degree of network disclosure; there was a significant correlation between the degree of disclosure and self-esteem of college students in the log reply and the number of the owner recovery log; the relationship between the number of friends, the number of log reply and the number of photos shows the relationship between the disclosure level and the mental health. Conclusions indicate that appropriate information disclosure has a good effect on the maintenance of mental health.
\end{abstract}

Keywords: Network, Self-Disclosure, Mental Health, Self-Esteem

\section{Introduction}

The characteristics of self-disclosure are a symbol of healthy personality, and it's the realization of interpersonal communication and maintaining a healthy psychological ability, playing an important role in interpersonal communication. The appropriate balance of privacy and disclosure is conducive to the normal development of interpersonal relations, and has an important role in the development of mental health.

The western research on self-disclosure is shown in many aspects, such as the relationship between self-disclosure and its influencing factors, self-disclosure and psychotherapy, self-disclosure and physical and mental health, etc. Lazarus pointed out that the treatment of the language of self-disclosure has opened up the efficacy of the treatment of communication channels [2]. Pennebaker and Petrie draw a conclusion through the experiment: in 6 months, subjects who spoke out depressive emotion had less chance to go to the health counseling center [3]. It can be seen that self-disclosure and people's mental health has a very close relationship, especially in the personal adaptation and psychological treatment. It plays an important role in.

At present in our country, college students as a special social group, psychological commitment to learning, employment, interpersonal relationships and other intangible social pressure. Social environment changes and their physical and psychological changes have deepened their 
psychological conflicts, which make them have more psychological and behavioral problems. At present, the college students' self-disclosure is transferred from the past face to face communication, to the Internet as the medium, instead of reality communication. Renren and his colleges, such social networking sites are well received by students, and become an important way for college students to express themselves and their friends.

The study shows that the construction of social interaction, self-image and the search for information are the three main motivations for college students to use social networking sites. Using social networking sites and real life social trust than demographic data can better predict the degree of self-disclosure on social networking sites. They can at any time through the network media to show the status of the moment, through the state, the log, upload photos and other acts to share their feelings, and even possible disclosure their personal privacy.

Mental health people can make proper self-disclosure in real life, so as to establish a close relationship to obtain good interpersonal communication; college students who can't set up good interpersonal support in real life, such as those who are introverted, poor communication, hope to get attention but also very lonely; someone who has had some setbacks in life; a child in an unharmonious family; the study result is not prominent, the mood is depressed, because the environment change causes the result to fall again and difficult to adapt, these youth may pay more attention to the interpersonal interaction on the Internet. The university stage is a continuation of the performance for the adolescent, closeness and openness co-exist, the university stage gradually sophisticated, so that young people closed his heart, show less to others, but at the same time they also feel very lonely, and hope someone can pay attention to and understand them. College students use the Internet more frequently, and participate in the content and form self-disclosure on the network. College students in the sense that their disclosure to the network is more appropriate, but the immature personality traits make them easy to rely on the network. Virtual social network platform can make up for the reality of loneliness, established friendship, but also has the potential to make it a sense of deviation from the real life. These social network platform provide a way to college students of self-disclosure, it will also bring some problems at the same time.

In China, the previous research mainly explores the self-disclosure of college students in face to face communication, well paid less attention to the self-disclosure on the network, and the discussion on the self-disclosure of the network has gradually become the focus of research on self-disclosure. A scale for measuring the network and the reality of self-disclosure has been compiled in China. The overall features used to investigate the overall characteristics of the network and the reality of self-disclosure of college students in China, used to explore the differences between college students' self-disclosure and real self-disclosure, and the relationship between self-disclosure and self-disclosure of the Internet and the sense of loneliness [4]; adolescent selfdisclosure questionnaire is designed to fully grasp the characteristics of adolescents' self-disclosure [5].

The study shows that the degree of self-disclosure of the network has a significant positive predictive effect on loneliness [4].

This study combined with the experience of teachers in school mental health education center. The key point is to explore the relationship between self-disclosure and self-esteem and mental health status of the network. Significance is to strengthen the guidance of college students' self-disclosure regulation, to enhance the sense of self psychological identity of college students, to reduce the deviation of the network and reality, so as to correctly use the network. Fundamentally improve college students' psychological adaptation ability and psychological quality, so as to guide college students to use the network to carry out self - disclosure, and promote the psychological health. We take this as a health assessment index of mental health in order to educate freshmen.

\section{Research Methods}

\subsection{Objects}

The objects is a random sample of 172 undergraduate students, including 99 men and 73 women; 27 freshman, 109 sophomores, 27 juniors, 9 seniors; 8 to 24 years old.

\subsection{Research Tools}

Use symptom self-rating scale SCL-90, self-esteem scale SES, general situation questionnaire, and network self-disclosure questionnaire.

\subsubsection{Symptom Self-rating Scale SCL-90}

The scale consisted of 90 items, with 1-5 scoring method, usually use to evaluation of various psychological symptoms and their severity in one week. A total of 9 subscales, namely: somatization, obsessive-compulsive symptoms, interpersonal sensitivity, depression, anxiety, hostility, phobia, paranoia and psychosis, and other additional projects. The higher score shows that the overall mental health level down, a factor of more than 2, indicating the very factor has serious psychological problems.

\subsubsection{Self-esteem Scale (SES)}

The scale consists of 10 items that are directly reported by the subjects to be consistent with their own. Grade Four score, 1 means consistent, 2 means accord, 3 means it did not meet, 4 means it was not consistent. The total score range is $10-40$ points, the higher the score, the higher the degree of self-esteem. This is designed to assess the individual's overall feelings of self-worth and self-acceptance.

\subsubsection{General Situation Questionnaire}

Include: name, gender, age, grade, family, home location, family situation, family income. 


\subsubsection{Network Self-disclosure Questionnaire}

Use Renren to do the information collection: including the number of friends, published log number, the number of the States, and friends reply to log and status, the owner return number, photo number, upload the number of photos oneself, expressions, such as smile, serious, pose, and sad [6].

\subsection{Data Processing}

All data is used SPSS18.0 software to input and statistical analysis.

\section{Research Results}

\subsection{The Relationship Between Mental Health and Self-esteem in the Investigation Group}

\subsubsection{The Basic Situation of Mental Health and Self-esteem in the Investigation Group}

The SCL-90 score of the research group was found to be compared with the norm: the mean value of the investigation group was higher than that of the norm, see table 1. Overall score of college students participating in the survey: the total score (M:25.65, SD:2.44) was higher than the average (self-esteem score from $10-40$ points, take 25 points to average).

Table 1. Comparison between study group and normal mode SCL-90 score $(X+S)$

\begin{tabular}{llll}
\hline & Norm & Research Group & t \\
\hline Somatic & $1.34 \pm 0.45$ & $1.53 \pm 0.51$ & $-4.51^{* *}$ \\
Force & $1.69 \pm 0.61$ & $2.08 \pm 0.70$ & $-6.76^{* *}$ \\
Interpersonal sensitivity & $1.76 \pm 0.67$ & $1.85 \pm 0.69$ & -1.55 \\
Depressed & $1.57 \pm 0.61$ & $1.68 \pm 0.62$ & $-2.11^{*}$ \\
Anxious & $1.42 \pm 0.43$ & $1.68 \pm 0.64$ & $-5.08^{* *}$ \\
Hostility & $1.50 \pm 0.57$ & $1.72 \pm 0.70$ & $-3.85^{* *}$ \\
Terror & $1.33 \pm 0.45$ & $1.49 \pm 0.55$ & $-3.56^{* *}$ \\
Delusion & $1.52 \pm 0.60$ & $1.70 \pm 0.57$ & $-3.71^{* *}$ \\
Mental illness & $1.36 \pm 0.47$ & $1.64 \pm 0.57$ & $-6.01^{* *}$ \\
\hline
\end{tabular}

Note: $* \mathrm{p}<0.001, * \mathrm{p}<0.01$

\subsubsection{The Relationship Between Mental Health and Self-esteem of College Students}

Table 2 shows that: the scores of college students' self-esteem is not significantly related to their total score of SCL-90, but it is significantly related to the hostility factor.

Table 2. Correlation between self-esteem and mental health and various factors of SCL-90.

\begin{tabular}{llllllllll}
\hline & $\begin{array}{l}\text { Total score of } \\
\text { mental health }\end{array}$ & Somatic & Force & $\begin{array}{l}\text { Interpersona } \\
\text { I sensitivity }\end{array}$ & Depre-ssed & Anxious & Hostility & Terror & Delusion \\
\hline $\mathrm{r}$ & 0.097 & 0.040 & 0.076 & 0.066 & 0.051 & 0.136 & $0.211 * *$ & 0.063 & 0.127 \\
\hline
\end{tabular}

Note: $* \mathrm{p}<0.001, * \mathrm{p}<0.01$

\subsubsection{Differences in Mental Health of High and Low self-esteem Groups}

According to the score of the self-esteem scale: take greater than the average for the high self-esteem group, lower than the average for the low self-esteem group (self-esteem score from 10-40 points, take 25 points into the average). The scores of SCL-90 factors and mental health scores were different, and there were significant differences between the two factors of anxiety and hostility, see table 3 .

Table 3. SCL-90 factors and mental health scores of high /low self-esteem group.

\begin{tabular}{llll}
\hline & $\begin{array}{l}\text { Low self-esteem } \\
\text { group }\end{array}$ & $\begin{array}{l}\text { High self-esteem } \\
\text { group }\end{array}$ & t \\
\hline $\begin{array}{l}\text { Total score of mental } \\
\text { health }\end{array}$ & $60.35 \pm 46.22$ & $68.65 \pm 53.31$ & -1.09 \\
Somatic & $1.50 \pm 0.46$ & $1.57 \pm 0.57$ & -0.86 \\
Force & $2.03 \pm 0.65$ & $2.13 \pm 0.76$ & -0.98 \\
Interpersonal & $1.83 \pm 0.67$ & $1.86 \pm 0.72$ & -0.27 \\
sensitivity & $1.65 \pm 0.61$ & $1.72 \pm 0.63$ & -0.68 \\
Depressed & $1.58 \pm 0.47$ & $1.79 \pm 0.79$ & $-2.21^{* *}$ \\
Anxious & $1.59 \pm 0.58$ & $1.89 \pm 0.80$ & $-2.86^{* *}$ \\
Hostility & $1.47 \pm 0.55$ & $1.51 \pm 0.55$ & -0.49 \\
Terror & $1.69 \pm 0.57$ & $1.71 \pm 0.58$ & -0.23 \\
Delusion & $1.61 \pm 0.56$ & $1.67 \pm 0.59$ & -0.70 \\
\hline Mental illness & & & \\
\hline
\end{tabular}

Note: $* \mathrm{p}<0.001, * \mathrm{p}<0.01$

\subsection{Investigation on the Degree of Internet Self-disclosure}

Network self-disclosure will participate in the survey of college students in Renren's operation behavior, including the number of friends, published log number, the number of the States, and friends reply to log and status, the owner return number, photo number, upload the number of photos oneself, expressions, such as smile, serious, pose, and sad, etc. Standard deviation of the large display network disclosure is not average, see table 4 .

Table 4. Self-disclosure data on Renren.

\begin{tabular}{lll}
\hline Statistics & Mean value & Standard deviation \\
\hline Number of friends & 295.1 & 256.532 \\
Status number & 41.49 & 63.322 \\
Status reply number & 180.48 & 521.621 \\
Owner reply number & 121.83 & 306.915 \\
Log number & 1.34 & 3.088 \\
Log reply number & 11.76 & 35.806 \\
Owner reply log number & 6.71 & 19.486 \\
Photos number & 5.72 & 8.155 \\
Upload photos number & 12.41 & 22.824 \\
Photo expression - serious & 2 & 4.262 \\
Photo expression - smile & 5.15 & 8.404 \\
Photo expression - pose & 2.85 & 4.136 \\
Photo expression - sad & 0.26 & 0.861 \\
\hline
\end{tabular}


The network self-disclosure data shows: there are gender differences in the extent of Internet self-disclosure, when upload photos, men and women had different numbers, there were significant differences in the expression of the photo, the smile, the pose, the sadness, see table 5:

Table 5. Correlation between gender and self-disclosure.

\begin{tabular}{|c|c|c|c|c|c|c|}
\hline & Number of friends & State number & Status reply number & Owner reply number & Log number & Log reply number \\
\hline male & $273 \pm 245.99$ & $1.42 \pm 3.30$ & $13.61 \pm 43.75$ & $6.79 \pm 22.35$ & $5.03 \pm 8.35$ & $9.25 \pm 21.35$ \\
\hline female & $323.55 \pm 269.27$ & $1.23 \pm 2.79$ & $9.26 \pm 20.69$ & $6.60 \pm 7.84$ & $6.66 \pm 7.84$ & $16.68 \pm 24.18$ \\
\hline $\mathrm{t}$ & -1.25 & 0.40 & 0.79 & -1.30 & -1.30 & $-2.13^{*}$ \\
\hline
\end{tabular}

Table 5. Continue.

\begin{tabular}{|c|c|c|c|c|c|c|c|}
\hline & Owner reply log number & Photos number & Upload photos number & serious & smile & pose & sad \\
\hline male & $40.05 \pm 69.06$ & $194.43 \pm 651.90$ & $117.70 \pm 353.50$ & $1.94 \pm 3.71$ & $2.98 \pm 5.29$ & $1.81 \pm 2.84$ & $0.04 \pm 0.32$ \\
\hline female & $43.45 \pm 55.00$ & $161.56 \pm 259.13$ & $127.42 \pm 231.46$ & $2.08 \pm 4.94$ & $8.08 \pm 10.07$ & $4.27 \pm 5.11$ & $0.55 \pm 1.21$ \\
\hline $\mathrm{t}$ & -0.35 & 0.41 & -0.20 & -0.22 & -4.11 & $-4.03 * *$ & $-3.98 * *$ \\
\hline
\end{tabular}

Note: $* \mathrm{p}<0.001, * \mathrm{p}<0.01$

There were significant correlation between the number of friends, the state, and the way of self-disclosure; there was a significant correlation between the $\log$ and the number of replies, the number of states, the number of owner reply, and the expression of sadness; there was a significant correlation between log replies with the owner reply log, the owner's head portrait, and others' photos, the status of the state, the owner reply, the owner is significantly related to reply; there was a significant correlation between owner reply log and the owner's head portrait, status, state reply, owner reply status, smile and sad face; there is a significant correlation between the the owner's head portrait and the state and the facial expression; there is a significant correlation between other photos and with a smile, pose, sad expression; there is also a correlation between expressions. See table 6:

Table 6. The relationship between network self-disclosures.

\begin{tabular}{|c|c|c|c|c|c|c|c|c|c|c|c|c|c|}
\hline & friends & $\log$ & $\begin{array}{l}\text { Log } \\
\text { reply }\end{array}$ & $\begin{array}{l}\text { owner } \\
\text { reply log }\end{array}$ & $\begin{array}{l}\text { owner's } \\
\text { head } \\
\text { portrait }\end{array}$ & $\begin{array}{l}\text { Upload } \\
\text { photos } \\
\text { number }\end{array}$ & status & $\begin{array}{l}\text { status } \\
\text { replay }\end{array}$ & $\begin{array}{l}\text { owner } \\
\text { reply } \\
\text { status }\end{array}$ & serious & smile & pose & sad \\
\hline friends & 1 & & & & & & & & & & & & \\
\hline $\log$ & $.364 * *$ & 1 & & & & & & & & & & & \\
\hline Log reply & $.323 * *$ & $.600 * *$ & 1 & & & & & & & & & & \\
\hline owner reply log & $.416^{* *}$ & $.614^{* *}$ & $.955^{* *}$ & 1 & & & & & & & & & \\
\hline $\begin{array}{l}\text { owner's head } \\
\text { portrait }\end{array}$ & $.448 * *$ & .114 & $.264 * *$ & $.292 * *$ & 1 & & & & & & & & \\
\hline $\begin{array}{l}\text { others head } \\
\text { portrait }\end{array}$ & $.159 *$ & .001 & -.044 & -.015 & .129 & 1 & & & & & & & \\
\hline status & $.456^{* *}$ & $.379 * *$ & $.234 * *$ & $.265 * *$ & $.349 * *$ & .053 & 1 & & & & & & \\
\hline status replay & $.301 * *$ & $.190 *$ & $.348 * *$ & $.331 * *$ & $.508 * *$ & -.007 & $.615^{* *}$ & 1 & & & & & \\
\hline owner reply status & $.411 * *$ & $.275^{* *}$ & $.367 * *$ & $.387 * *$ & $.492 * *$ & .000 & $.693 * *$ & $.960 * *$ & 1 & & & & \\
\hline smile & $.502 * *$ & .132 & .118 & $.205^{* *}$ & $.591 * *$ & $.376^{* *}$ & $.377 * *$ & $.279 * *$ & $.350 * *$ & $.247 * *$ & 1 & & \\
\hline pose & $.383 * *$ & -.019 & .012 & .080 & $.369^{* *}$ & $.317 * *$ & $.216^{* *}$ & .092 & .137 & $.493 * *$ & $.487 * *$ & 1 & \\
\hline sad & $.294 * *$ & $.209 * *$ & $.183^{*}$ & $.280 * *$ & $.450 * *$ & $.170^{*}$ & $.150^{*}$ & $.226^{* *}$ & $.283^{* *}$ & $.355^{* *}$ & $.477 * *$ & $.329 * *$ & 1 \\
\hline
\end{tabular}

Note: $* \mathrm{p}<0.001, * \mathrm{p}<0.01$

\subsection{The Relationship Between Self-esteem and Self-disclosure of the Internet}

The total score of self-esteem was significantly related to the number of log reply and the number of master replies.

There were significant correlation between expression and serious. There were correlation between high self-esteem group and the number of friends, log number, and others' photos, and there were significant correlation between high self-esteem group and the number of log reply, the number of owner replies log and the serious facial expressions, there was no significant correlation between the self-esteem and the self-disclosure on the Internet. See table 7. 
Table 7. Relationship between self-esteem and self-disclosure of the network.

\begin{tabular}{llll}
\hline & Total score of self-esteem & High score group & Low score group \\
\hline Number of friends & 0.117 & $0.250^{*}$ & -0.119 \\
Log number & 0.022 & $0.277^{*}$ & 0.048 \\
Log reply number & $0.232^{* *}$ & $0.345^{* *}$ & 0.079 \\
Owner reply log number & $0.213^{* *}$ & $0.337^{* *}$ & 0.031 \\
Status number & -0.048 & 0.109 & 0.073 \\
Status reply number & -0.063 & -0.054 & -0.052 \\
Owner reply Status number & -0.055 & -0.046 & 0.006 \\
Owner's head portrait & 0.117 & 0.058 & -0.107 \\
Upload photos number & 0.003 & $0.233^{*}$ & -0.012 \\
Photo expression - serious & $0.237^{* *}$ & $0.367^{* *}$ & 0.062 \\
Photo expression - smile & -0.071 & 0.054 & -0.137 \\
Photo expression - pose & 0.099 & 0.197 & -0.134 \\
Photo expression - sad & 0.037 & $0.229^{*}$ & -0.178 \\
\hline
\end{tabular}

Note: $* \mathrm{p}<0.001,{ }^{*} \mathrm{p}<0.01$

The average self-disclosure of high - esteem group was higher than that of low group, but the T value was not significantly different. See table 8 .

Table 8. Independent samples T test of self-disclosure in self-esteem group and network.

\begin{tabular}{llll}
\hline & Low self-esteem group & High self-esteem group & t \\
\hline Number of friends & $280.12 \pm 250.774$ & $313.17 \pm 263.794$ & -0.840 \\
Log number & $1.63 \pm 3.504$ & $1.00 \pm 2.476$ & 1.330 \\
Log reply number & $9.85 \pm 20.380$ & $14.06 \pm 48.329$ & -0.767 \\
Owner reply log number & $5.64 \pm 13.903$ & $8.00 \pm 24.631$ & -0.790 \\
Status number & $48.97 \pm 75.647$ & $32.49 \pm 42.925$ & 1.709 \\
Status reply number & $200.89 \pm 613.627$ & $155.88 \pm 385.158$ & 0.562 \\
Owner reply Status number & $138.35 \pm 361.360$ & $101.91 \pm 225.257$ & 0.774 \\
Owner's head portrait & $4.59 \pm 6.899$ & $7.09 \pm 9.313$ & -2.023 \\
Upload photos number & $14.07 \pm 26.583$ & $10.40 \pm 17.202$ & 1.052 \\
Photo expression - serious & $1.68 \pm 3.614$ & $2.38 \pm 4.929$ & -1.079 \\
Photo expression - smile & $5.61 \pm 9.890$ & $4.59 \pm 6.176$ & 0.789 \\
Photo expression - pose & $2.54 \pm 4.287$ & $3.23 \pm 3.941$ & -1.087 \\
Photo expression - sad & $0.24 \pm 0.947$ & $0.27 \pm 0.750$ & -0.186 \\
\hline
\end{tabular}

\subsection{Research on Mental Health and Self-disclosure of Internet}

SCL-90 evaluation results scores and the number of disclosure information being investigated on their personal home page to do Spearman nonparametric correlation coefficient analysis. There was a significant negative correlation between the number of good friends and mental health factors in the paranoia, interpersonal sensitivity, anxiety and terror; there was a significant negative correlation between friends who published some of the text in the owner page for a reply and interpersonal sensitivity, anxiety; there was a significant negative correlation between the smiling expression of photos and mental illness, delusion, somatic, obsessive, relationship sensitive, depression, anxiety and terror; however, there was a significant correlation between the serious expression and the physical, anxiety and hostility. See table 9.

Table 9. The correlation coefficient between the total score of mental health, the factors and the contents of the disclosure.

\begin{tabular}{|c|c|c|c|c|c|c|}
\hline & $\begin{array}{l}\text { Number of } \\
\text { friends }\end{array}$ & $\begin{array}{l}\text { status reply } \\
\text { number }\end{array}$ & $\begin{array}{l}\text { Owner reply } \\
\text { number }\end{array}$ & Serious & Smile & $\begin{array}{l}\text { Total reply } \\
\text { number }\end{array}$ \\
\hline Total score of mental health & -0.14 & -0.101 & -0.117 & 0.15 & $-.265 * *$ & -0.12 \\
\hline Somatic & -0.115 & -0.134 & -0.114 & $.194 *$ & $-.162 *$ & -0.138 \\
\hline Interpersonal Sensitivity & $-.159^{*}$ & $-.150^{*}$ & $-.154 *$ & 0.102 & $-.286 * *$ & $-.159 *$ \\
\hline Depressed & -0.09 & -0.059 & -0.086 & 0.115 & $-.236^{* *}$ & -0.098 \\
\hline Anxious & -0.133 & $-.162 *$ & $-.160 *$ & $.172 *$ & $-.301 * *$ & $-.166^{*}$ \\
\hline Hostility & $-.176^{*}$ & -0.034 & -0.021 & $.155^{*}$ & -0.111 & -0.052 \\
\hline Delusion & $-.188^{*}$ & -0.114 & -0.128 & 0.135 & $-.319 * *$ & -0.111 \\
\hline Mental illness & -0.055 & -0.102 & $-.156^{*}$ & 0.145 & $-.249 * *$ & -0.131 \\
\hline Terror & $-.206^{* *}$ & -0.065 & -0.081 & 0.106 & $-.315 * *$ & -0.081 \\
\hline
\end{tabular}

Note: ${ }^{*} \mathrm{p}<0.001, * \mathrm{p}<0.01$ 


\section{Discussion}

\subsection{Relationship Between Self-esteem and Mental Health}

Self-esteem is a person's self recognition and self worth, it covers self satisfaction and self value. It reflects the individual's own characteristics of the evaluation, feelings and attitudes, indicates how much individuals believe they are capable, important, successful, and valuable [7]. Self-esteem is the evaluation of self concept, which is the evaluation of the individual's positive or negative self. In previous studies, the close relationship between self-esteem and mental health has been repeatedly verified. High self-esteem and health related to social requirements of things (such as optimism, successful reply, positive emotions, physical health, etc.) [8]. Yang Lizhu pointed out that self-esteem is an important indicator of mental health [9]; Chen Jianwen's research also showed that the majority of college students' self-esteem and SCL-90 score was significantly negatively related to [10]; Hu Junsheng's study further shows that high self-esteem individuals not only have a higher level of mental health and self harmony, but also has inhibition behavior and fewer psychosomatic symptoms, and it has a positive effect on psychological health of college students self-esteem [11].

High hostility shows that this person's self defense ability is better, strong sense of self, so it is closely related to self-esteem. There was a significant correlation between self-esteem and mental health, Hostility and self-esteem were significantly related. With higher hostility, the level of self-esteem is higher. There was a significant difference between the level of mental health and self-esteem. In addition, the influence of anxiety and hostility on self-esteem is significant. For college students, anxiety and hostility are worth noticing. They have a significant effect on the structure of college students' self-esteem.

\subsection{Network Self-disclosure Analysis}

Network self-disclosure data shows: there were differences between the genders in the degree of self-disclosure on the Internet, female has more friends on the internet, they are more willing to upload photos to show themselves, and they are also more willing to show a variety of expressions.

Domestic related research also shows: there is a significant gender difference in college students' network and real life about self-disclosure, the total score about self-disclosure of parent-child relationship, heterosexual interaction, emotional dimension on the internet, the male students were significantly higher than female students; the total score about self-disclosure of study, emotion, personality, attitude, the male students are significantly lower than female students [4].

The number of friends, the degree of elf-disclosure, and the degree of interaction are positive correlation with others. This reflected a good social support, that is, through the virtual communication to get the outside world to understand, give, so that to meet the need particularly the mental need [12]. A lot of research on adult [13] [14] [15] shows that "obtaining social support" is one of the main motivations of virtual communication; Gustavo's study on the Israeli youth's virtual communication [16], the domestic research on the motivation of college students are also shown: in the face of family conflict, the reality of the pressure, young people tend to have more network communication, that is, to find social support [17] [18].

\subsection{The Relationship Between Self-esteem and Self-disclosure of the Network}

People with high self-esteem related to the number of friends are more, the number of logs is more, and the number of self-timer photos is high. Interacting much with friends on social networks, that is, people with good interpersonal support, self-esteem is relatively high. High self-esteem is also associated with a serious expression. High self-esteem usually show in a more rigorous and serious attitude to in front of friends.

\subsection{The Relationship Between Internet s Self-disclosure and Mental Health}

The number of friends on the social networking site was associated with a number of psychological factors. To a certain extent, the statistical results indicate that the increase of the number of friends has a retard effect on the psychological problems caused by the above reasons. House owner publish a lot of information in home page in the hope that their friends can see and get the feedback. The more times the information is returned, the more it helps to eliminate the sensitivity of the owner to the interpersonal relationship, and to ease the anxiety. When a friend received a reply to his message, the owner will selectively give feedback. From the statistical results, the number of reply to owner and interpersonal sensitivity, anxiety, mental illness was negatively correlated. It can be inferred that the more information the owner to friends reply, the less chance of anxiety and mental illness the owner may haves. As the saying goes "ten years younger with smile", smiling much is a visible improvement in the overall level of mental health. So the results show that: individual upload more smile photos has less psychological problems. "Worry a lot bring a white head", always being a serious expression or laughing less will increase the body's disease. Psychological problems of anxiety may also increase the hostility to other things, and more aggressive.

\subsection{Educational Advice}

According to "the 2009 Chinese Internet users Social Network Application Research Report" core data, students are the main users of social networking sites. Nearly $80 \%$ of the social networking site users said their network friends are from real life. Users of social networking sites are the most commonly used communication and self-expression function. 
$60 \%$ user's information is true. Nearly $80 \%$ of users believe that social networking sites help themselves to maintain a relationship. $50 \%$ users of social networking sites will add friends in reality to the network instant messaging software. $90 \%$ users believe that the use of social networking sites have no effect on social reality.

Indeed, the Internet brings a lot of convenience and support to people's life, but the network security cannot be underestimated. Casual self-disclosure to the Internet is likely to reveal personal information, excessive self-disclosure to privacy may also cause unnecessary damage. Therefore, we should advocate college students' health and safety about the network self-disclosure.

College students can conduct moderate self-disclosure on the Internet, so as to raise the level of psychological health. Under the premise of the protection of personal privacy, reasonable, appropriate self-disclosure is conducive to ease the pressure, maintain good interpersonal relationships, to give the individual a certain social support.

\section{Conclusions}

Self-esteem and mental health of the hostility factor showed significant correlation. Male and female college students have significant differences in the degree of network disclosure. There was a significant correlation between the degree of self-disclosure and self esteem of college students in the log reply number and the owner reply log. There was a significant correlation between the the disclosure level of friends number, log reply number, photos number and the mental health.

\section{Acknowledgments}

This work was supported by National Science Foundation of China (DUT16rw001) and Liaoning Province Education Science "12th Five-Year" planning college students ideological and political education special (JGZXS13039).

\section{References}

[1] Jourard, S. M. Some factors in (1). the Journal of Abnormal and Social Psychology, 1958, 56, 91-98.

[2] Lazarus, A: Behavior Therapy and Beyond, New York, McGraw Hill, 1971.

[3] Pennebaker J. W. \& Howver, C. W: Inhibition and cognition: Toward an understanding of trauma and disease. In R. J. Davidson, G.. E Schwartz, \& D. Shapiro (Eds. ),Consciousness and self - regulation, 1986, 4,107-136.

[4] Yu Minglu. The scale of College Students' network and the reality of self disclosure and its relationship with loneliness [D] Hebei: Hebei Normal University, 2011:1-1.

[5] Yang Fanglin. The development of adolescent self disclosure questionnaire and its relationship with loneliness [D]. Wuhan: Huazhong Normal University, 2011:1-1.

[6] Andersson PK, Gustafsson A, Kristensson P, Wästlund E. The effect of frontline employees' personal self-disclosure on consumers' encounter experience. Journal of Retailing and Consumer Services. 2016; 30: 40-9.

[7] Green T, Wilhelmsen T, Wilmots E, Dodd B, Quinn S. Social anxiety, attributes of online communication and self-disclosure across private and public Facebook communication. Computers in Human Behavior. 2016; 58: 206-13.

[8] Huang H-Y. Examining the beneficial effects of individual's self-disclosure on the social network site. Computers in Human Behavior. 2016; 57: 122-32.

[9] Kim J, Song H. Celebrity's self-disclosure on Twitter and parasocial relationships: A mediating role of social presence. Computers in Human Behavior. 2016; 62: 570-7.

[10] Knop K, Öncü JS, Penzel J, Abele TS, Brunner T, Vorderer P, et al. Offline time is quality time. Comparing within-group self-disclosure in mobile messaging applications and face-to-face interactions. Computers in Human Behavior. 2016;55, Part B:1076-84.

[11] Lin R, Utz S. Self-disclosure on SNS: Do disclosure intimacy and narrativity influence interpersonal closeness and social attraction? Computers in Human Behavior. 2017;70:426-36.

[12] Wang De McLean M, Steinauer J, Schmittdiel J, Chan P, Dehlendorf C. Provider self-disclosure during contraceptive counseling. Contraception. 2017;95(2):161-6.

[13] Katelyn Y. A. Mckenna, Amie S. Green. Virtual Group Dynamics. Group Dynamics: Theory, Research, and Practice, 2002, 6(1): 116-127.

[14] Uwe Matzat. Cooperation and Community on the Internet: Past Issues and Present Perspectives for theoretical- empirical Internet Research. Analyse \& Kritik, 2004, 26(1):63-90.

[15] McKay Johnston. Netting Citizens. Scotland: Saint Andrew Press, 2004.54-80.

[16] Gustavo S. Mesch, Ilan Talmud. Making Friends Online: Personal Needs and Social Compensation. Paper presented at "Computer Networks and Social Networks: An International Conference”. University of Haifa, 2003. 25-27.

[17] Ma Ning. Analysis of the psychological mechanism of College Students' network communication. Guangxi Social Science, 2005,01: 190-192.

[18] Lai Haixiong, Guo Jianzhong. An analysis of online communication and interpersonal communication barriers of college students. China Electric Power Education, 2002, 01:40-65. 